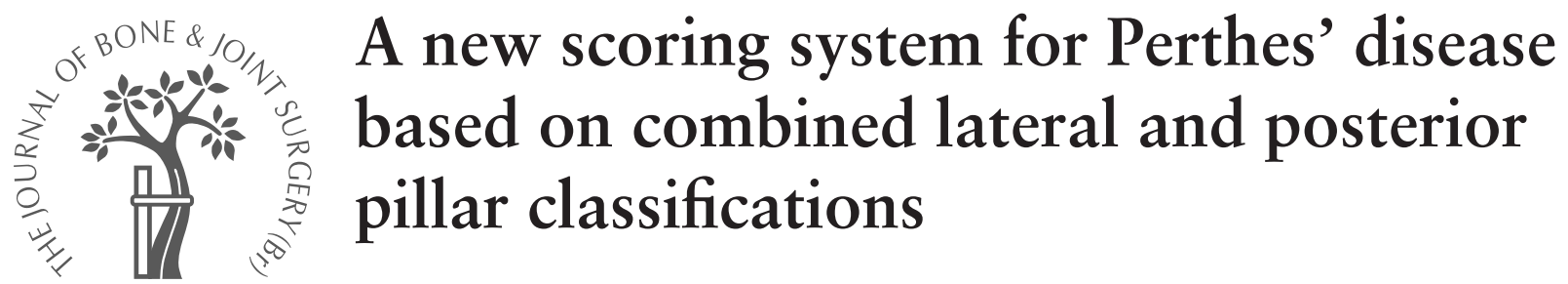

\author{
Y. Sugimoto, \\ H. Akazawa, \\ Y. Miyake, \\ S. Mitani, \\ K. Asaumi, \\ K. Aoki, \\ H. Inoue
}

\section{From Ehime}

Disabled Children's

Hospital, Ehime and

Okayama University,

Okayama, Japan

Y. Sugimoto, $\mathrm{MD}$

Orthopaedic Surgeon

H. Akazawa, MD, PhD,

Orthopaedic Surgeon

S. Mitani, MD, PhD,

Orthopaedic Surgeon

K. Asaumi, MD, PhD,

Orthopaedic Surgeon

K. Aoki, MD, PhD,

Orthopaedic Surgeon

$\mathrm{H}$. Inoue, MD, PhD,

Professor

Science of Functional

Recovery and

Reconstruction, Department

of Orthopaedic Surgery,

Okayama University

Graduate School of Medicine

and Dentistry, 2-5-1 Shikata-

cho, Okayama 700-8558,

Japan.

Y. Miyake, MD, PhD,

Orthopaedic Surgeon

Ehime Disabled Children's

Hospital, 7-2 Honmachi,

Matsuyama, Ehime 790

0811, Japan.

Correspondence should be sent to $\mathrm{Dr} \mathrm{H}$. Akazawa.

C2004 British Editorial

Society of Bone and

Joint Surgery

doi:10.1302/0301-620X.86B6.

$14622 \$ 2.00$

$J$ Bone Joint Surg [Br]

2004;86-B:887-91.

Received 13 May 2003;

Accepted after revision

17 September 2003

\title{
A new scoring system for Perthes' disease based on combined lateral and posterior pillar classifications
}

\begin{abstract}
We have devised a combined pillar score (CPS) system, based on the lateral pillar (LP) and the posterior pillar (PP) classifications, together with the age at onset of Perthes' disease, and examined its correlation with prognosis. The correlation coefficient of the Catterall classification, LP, PP, and CPS systems with the Stulberg system was $0.39,0.52,0.50$, and 0.70 , respectively. Overall 21 of the 22 hips $(95.4 \%)$ with a CPS of 0 to 1 point had a good outcome and 12 of the 13 hips (92.3\%) with a CPS of 3 points or more had a fair or poor outcome. None with a CPS of 2 points, had a poor outcome. The study shows that an accurate prediction of the prognosis is not possible with the LP classification alone for patients classified as belonging to group B (LP height $50 \%$ to $100 \%$ of contralateral height). The CPS system does allow accurate prediction of outcome.
\end{abstract}

A number of radiological classifications of Perthes' disease have been reported and are widely used in clinical practice. ${ }^{1-4}$ The Catterall classification quantifies the extent of epiphyseal involvement on anteroposterior (AP) and lateral radiographs. This classification has been widely used to assess the response to treatment, ${ }^{2,5}$ however, it has been reported to show not only a low inter-observer reliability, but also little correlation with the radiological outcome. ${ }^{6-10}$ The Salter-Thompson classification $^{2,11}$ is considered to be a useful and simple predictor of the prognosis, but in view of the incidence of subchondral bone fracture, it is not suitable as a basis for comparison. 3,9,12 The lateral pillar (LP) classification, which divided hips into three groups (A, $\mathrm{B}$ and $\mathrm{C}$ ) based on the height of the LP of the femoral head clearly predicted the radiological outcome, and appeared to be reliable. ${ }^{3,8-10,13,14}$ The LP group B, which includes more than half of the patients lacked discrimination. ${ }^{9,10,12}$ To refine discrimination in this group, Akazawa et $\mathrm{al}^{12}$ devised a posterior pillar (PP) classification, which assessed lateral radiographs. They reported on the usefulness of combining the LP with the PP classifications. We have devised a combined pillar score (CPS) system, based on the LP and PP classifications, and age at onset. The purpose of our study was to examine its correlation with the prognosis of Perthes' disease while studying the inter-observer and intra-observer reliability.

\section{Patients and Methods}

The study group comprised 56 hips in 56 patients with Perthes' disease treated at the Ehime Disabled Children's Hospital between 1975 and 1995. Patients with bilateral involvement and those who had received initial treatment at other hospitals were excluded. There were 51 boys and five girls, with 27 right and 29 left hips. The mean age at onset was 7.5 years $(2.8$ to 13.3$)$, the mean time from the onset until hospitalisation was 3.2 months $(0.1$ to 12.5), and the mean length of hospitalisation was 12.1 months (4 to 17). The mean age at the time of examination was 17.9 years (13.0 to 26.7) and the mean follow-up period was 11 years (3.5 to 18.8). All the patients were managed conservatively with skin traction following the same protocol which we have used during the past 20 years.

Analysis of predictive value. The radiographs taken during the fragmentation stage were assessed using the classifications of Catterall, ${ }^{1}$ Herring et al, ${ }^{3}$ Akazawa et al, ${ }^{12}$ the LP and the PP. The radiological outcome at the time of final examination was analysed according to the Stulberg system, ${ }^{15}$ with class I and class II being rated as good, class III as fair, class IV, and class $\mathrm{V}$ as poor. To avoid any bias in the assessment of the films, this was done simultaneously by two orthopaedic surgeons (HA and YS).

The PP classification assesses the posterior $15 \%$ to $30 \%$ of the femoral epiphysis according to the same criteria as those of the LP classification. ${ }^{3}$ Lateral radiographs taken during 


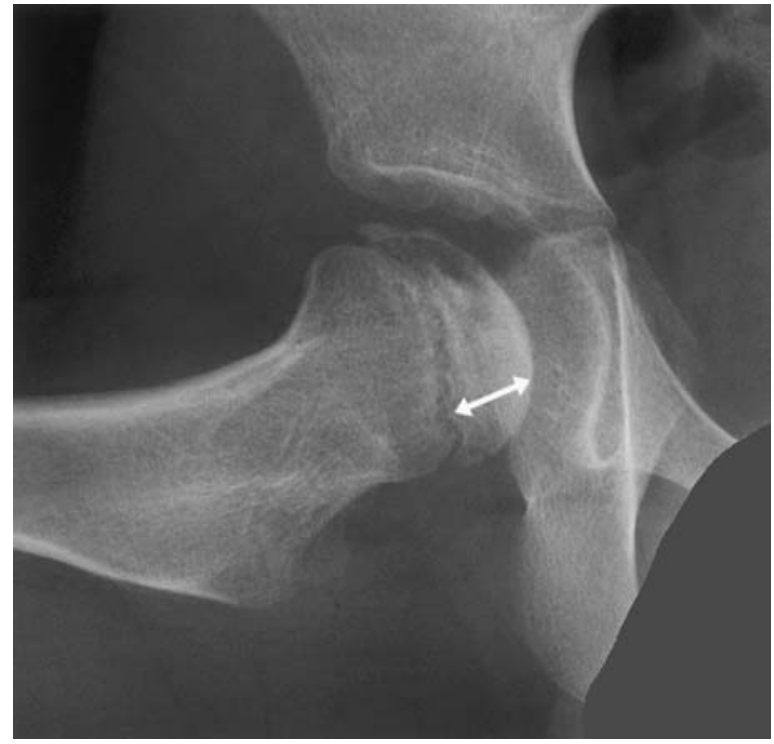

Fig. 1a

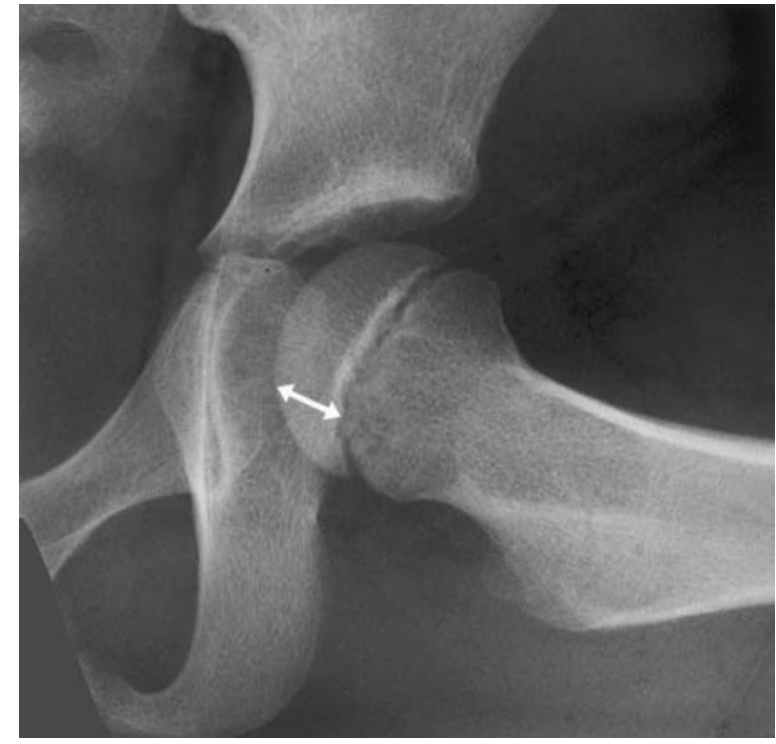

Fig. 1b

Radiographs for PP group A with a) showing that the posterior column is radiologically normal, and full height is maintained and b) the contralateral hip.

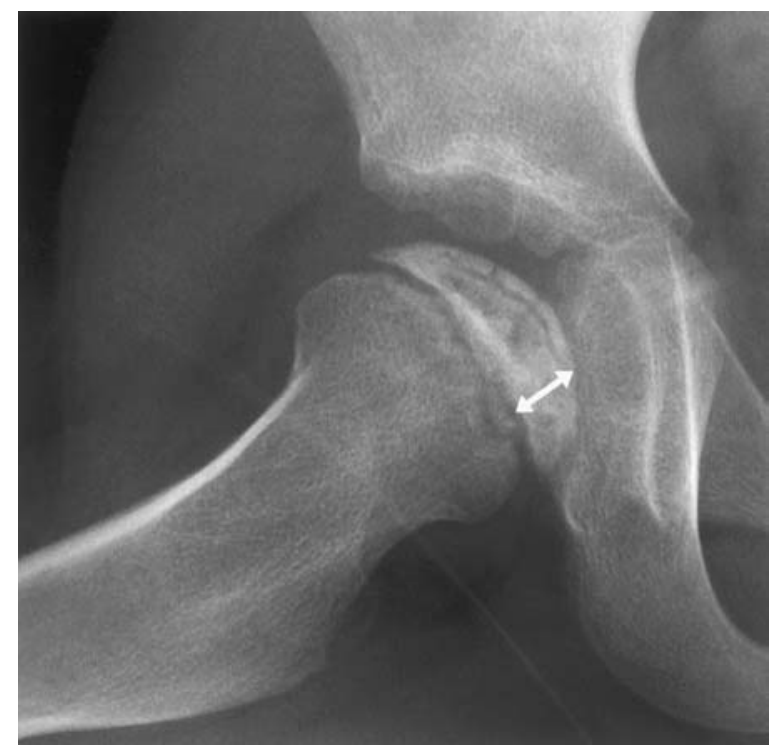

Fig. $2 a$

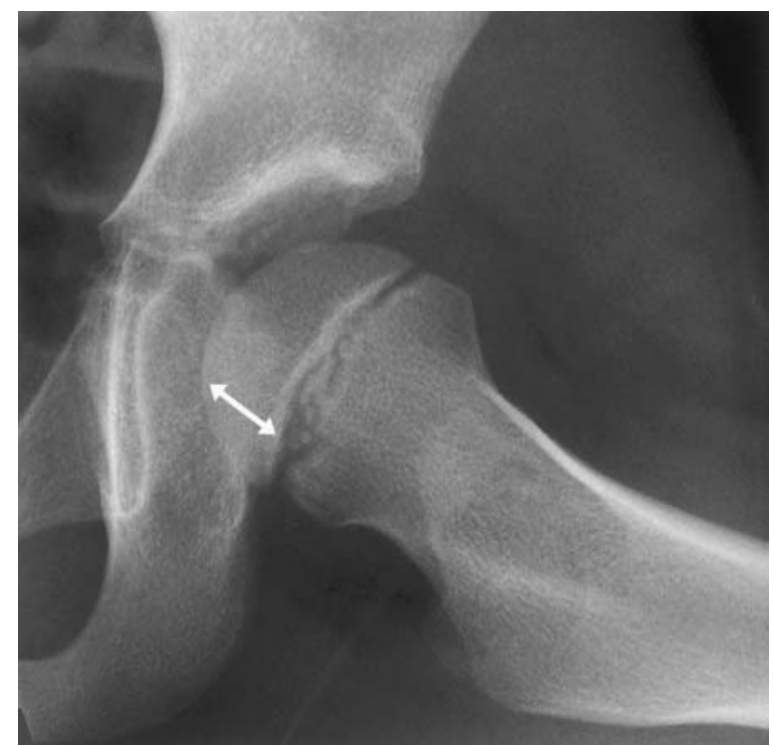

Fig. $2 b$

Radiographs for PP group B with a) showing that the height of the posterior column is between $50 \%$ and $100 \%$ of the original height and b) the contralateral hip.

the fragmentation stage were divided into three groups $(\mathrm{A}$, $\mathrm{B}$, and $\mathrm{C}) .^{3}$ In group $\mathrm{A}$, the PP and LP were radiologically normal; full height was maintained when compared with the contralateral hip. In group B, the heights of the PP and LP were between $50 \%$ and $100 \%$ of the contralateral height while in group $\mathrm{C}$ the heights were less than $50 \%$. For
PP, lateral radiographs were taken with the hip joints in $90^{\circ}$ of flexion and $45^{\circ}$ of abduction as described by Sugioka, Katsuki and Hotokebuchi, ${ }^{16}$ so that the pelvis and proximal femur are symmetrical (Figs 1 to 3 ).

The Catterall, LP, and PP classifications were examined for a correlation with the Stulberg system. Based on several 


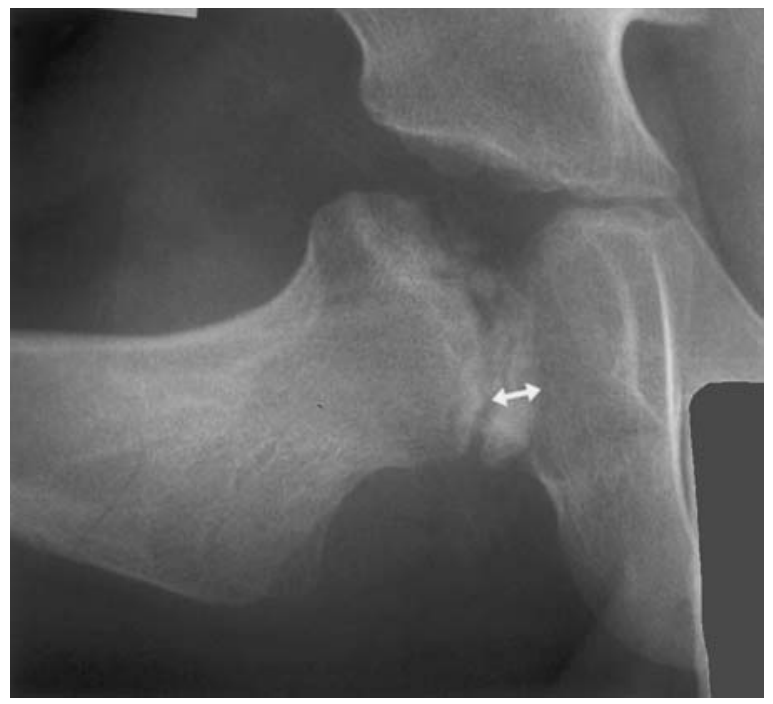

Fig. 3a

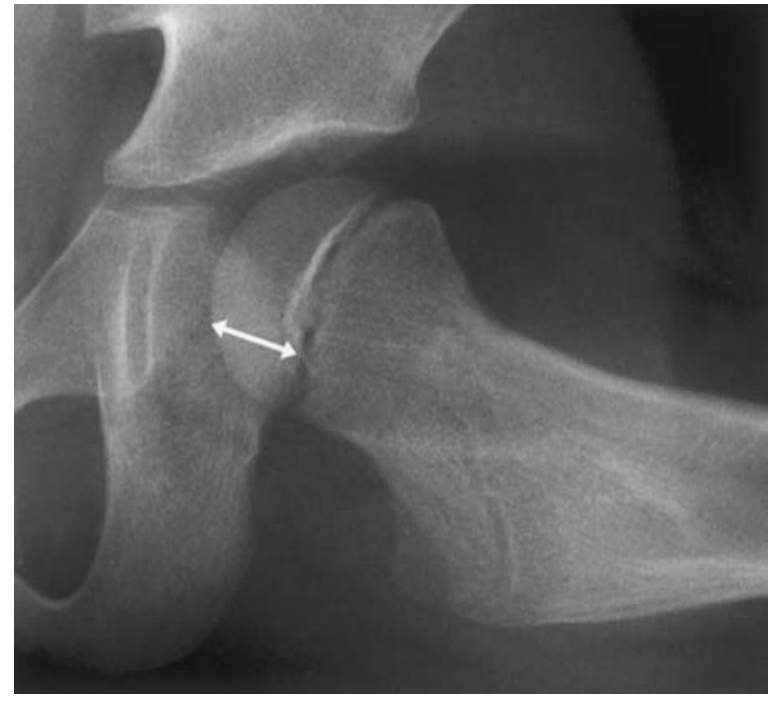

Fig. 3b

Radiographs for PP group C with a) showing that the height of the posterior column is less than $50 \%$ and b) the contralateral hip.

studies, the age at onset was used to divide the patients into two groups, one before and one after the age of nine years. ${ }^{3,10,13}$ Spearman's rank correlation coefficient was calculated to express the correlation of each datum with the Stulberg system. The correlation coefficient gives values between 0 to 1 , and a value of 1 indicates perfect correlation.

Inter- and intra-observer reliability were assessed using the method described by Podeszwa et al. ${ }^{14}$ We randomly selected 40 of the 56 patients for analysis; four orthopaedic surgeons with different levels of experience, assessed the lateral radiographs of the patients taken during the fragmentation stage, applying the PP classification; two of the four observers (YT and HA) were attending orthopaedic surgeons with more than ten years experience in paediatric orthopaedics, and the other two (SY and YS) had less than three years experience. To evaluate intra-observer reliability, at least 24 hours separated the first and second assessments. The results of the first assessment were used for evaluation of inter-observer reliability. Each observer also made an assessment of the AP radiographs according to the LP classification and the results were compared with those obtained using the PP classification.

The observed agreement, Cohen's kappa coefficient, ${ }^{17}$ and weighted kappa coefficient ${ }^{18}$ were calculated to assess statistically the inter- and intra-observer reliability of the classifications. The kappa and weighted kappa values were calculated using a five-point scale that ranged from 'slightly' to 'excellent' according to the guidelines of Landis and Koch. ${ }^{19}$

\section{Results}

According to the Catterall classification, there were no hips in group 1, but 21 hips in group II, 32 in group III, and three in group IV. The three hips classified in group IV belonged to PP group C. Of the 15 hips classified into PP group A, 13 (86.7\%) belonged to Catterall group II.

Following the LP classification, there were 18 hips in group A, 32 in group B, and six in group $C$ whereas under the PP classification, there were 15 hips in group A, 35 in group B, and six in group C; 23 of the 56 hips (41.1\%) were classified into different groups by the PP and LP classifications.

Based on the Stulberg system of radiological outcome, 24 hips were classified to class I, ten to class II, 18 to class III, four to class IV, and none to class V. Of the 21 hips classified into Catterall group II, $18(85.7 \%)$ had a good outcome, while all three in group IV were graded fair. The results for the hips in Catterall group III varied considerably between Stulberg class I and IV. Of 18 hips classified into LP group A, 16 (88.9\%) had a good radiological outcome, while the six hips classified into LP group $\mathrm{C}$ were fair or poor. Of all the 56 hips, $32(57.1 \%)$ were classified into LP group B, with varying outcomes between Stulberg class I and IV. To predict the outcomes based on the LP classification alone proved difficult. By contrast, all 15 hips classified into PP group A had a good outcome, but five of the six hips $(83.3 \%)$ classified into PP group C had a fair outcome. We classified 35 hips $(62.5 \%)$ into PP group B. Their outcomes varied between Stulberg class I and IV. Of the 43 hips in patients with an age at onset of less than nine years, 30 $(69.8 \%)$ had a good radiological outcome, but nine of the remaining $13(69.2 \%)$ with an age at onset of nine years or over had a fair or poor outcome. Calculation of Spearman's correlations between the Catterall, LP, and PP classifications, and the Stulberg system gave coefficients of 0.39 , 0.52 and 0.50 , respectively (Table I). 
Table I. Spearman's correlation coefficient between Stulberg classification and prognostic factors (LP, PP, CPS)

\begin{tabular}{ll}
\hline Factor & $\begin{array}{l}\text { Spearman's correlation } \\
\text { coefficient }\end{array}$ \\
\hline Catterall & 0.39 \\
Lateral pillar (LP) & 0.52 \\
Posterior pillar (PP) & 0.50 \\
Combined pillar score (CPS) & 0.70
\end{tabular}

Table II. Stulberg classification related to combined pillar score (CPS) for 56 hips

\begin{tabular}{lrrrrr}
\hline \multirow{2}{*}{$\begin{array}{l}\text { Combined } \\
\text { pillar score }\end{array}$} & \multicolumn{2}{c}{ Stulberg } & & & \\
\cline { 2 - 5 } & \multicolumn{1}{l}{ I } & II & III & IV & Total \\
\hline 0 & 6 & - & - & - & 6 \\
1 & 10 & 5 & 1 & - & 16 \\
2 & 8 & 4 & 9 & - & 21 \\
3 & - & 1 & 2 & 3 & 6 \\
4 & - & - & 6 & 1 & 7 \\
5 & - & - & - & - & 0 \\
Total & 24 & 10 & 18 & 4 & 56 \\
\hline
\end{tabular}

We chose the LP and PP classifications, which showed better coefficients than the Catterall classification, and combined them with the age at onset, to create the CPS in which hips were classified into groups A, B or C. Affected hips in LP and PP classifications were assigned a score of 0 , 1 , or 2, while an age at onset of less than nine years was given a score of 0 and an age of nine years or over was given a score of 1 . The sum of these scores, the CPS had a value between 0 and 5 .

In our study, 21 of the 22 hips $(95.4 \%)$ with a CPS of 0 to 1 point had a good outcome and 12 of the 21 hips $(57.1 \%)$ with a CPS of 2 points had a good outcome. None was graded as poor; 12 of the 13 hips $(92.3 \%)$ with a CPS of 3 or 4 points had a fair or poor outcome. None received a CPS of 5 points (Table II). Spearman's correlation coefficient was 0.70 between the CPS system and the Stulberg system, which was the highest among all the methods tested in this study (Table I).

With regard to the inter-observer reliability, the mean observed agreement using the PP classification was $75 \%$ (65 to 83 ). The mean kappa value was 0.55 (0.40 to 0.67 ) and the mean weighted kappa value was 0.65 (0.54 to 0.74), reflecting 'moderate' or 'substantial' agreement, respectively. The mean observed agreement using the LP classification was $76 \%$ (73 to 88 ). The mean kappa value was 0.56 (0.47 to 0.78$)$ and the mean weighted kappa value was 0.64 (0.56 to 0.82 ), reflecting moderate or substantial agreement, respectively.

Assessing the intra-observer reliability of the PP classification, the mean agreement was $91 \%$ (80 to 100). The mean kappa value was $0.82(0.65$ to 1.00$)$ and the mean weighted kappa value was 0.86 (0.73 to 1.00$)$, both of which reflected excellent agreement. Under the LP classification, the mean observed agreement was $87 \%$ ( 85 to 88 ).
The mean kappa value was 0.74 (0.68 to 0.76$)$ and the mean weighted kappa value was 0.78 (0.72 to 0.81$)$, both of which reflected 'substantial' agreement.

\section{Discussion}

Our study demonstrates that the LP and PP classifications correlated well and more closely with the radiological outcome, than did the Catterall classification. Spearman's correlations between the LP, PP and Catterall and the Stulberg system, gave coefficients of $0.52,0.50$, and 0.39 , respectively. Ritterbusch et $\mathrm{al}^{8}$ assessed 71 hips of patients with Perthes' disease using the Catterall and LP classifications. They found a correlation with the Stulberg system of 0.38 for the Catterall and 0.64 for the LP, suggesting that the LP classification was a more reliable predictor of the radiological outcome. Ismail and Macnicol $^{9}$ assessed 81 hips in 73 patients with Perthes' disease using the Catterall, SalterThompson and LP classifications together with arthrography to predict the prognosis. They reported that the LP and arthrography most closely correlated with the Stulberg system (the correlation coefficients were 0.57 and 0.53 , respectively). ${ }^{9}$ From these results, the LP classification was considered to be the simplest and most reliable method for classifying Perthes' disease.

One disadvantage of the LP classification is that prediction is difficult for group $\mathrm{B}$, into which more than half of patients fall. ${ }^{9,10,12}$ In our study, 32 of 56 hips $(57.1 \%)$ were classified into LP group B, and these hips had a variety of outcomes ranging from Stulberg class I to IV. Ismail and Macnicol $^{9}$ also reported that 48 of the 81 hips $(59.3 \%)$ classified into LP group B, had varied outcomes (13 hips were class I, 22 were class II, 12 were class III, and one was class IV). Our results suggest that accurate prediction of the prognosis is not possible with the LP classification alone for patients classified into group B.

The CPS system, however, allows accurate prediction for such patients. Of the 22 hips with CP scores of 0 to 1 point, $21(95.4 \%)$ had a good outcome and 12 of the 13 hips $(92.3 \%)$ with a CPS of 3 points or higher, had a fair or poor outcome. None of the hips with a CPS of 2 points had a poor outcome. The CPS system had the strongest correlation with the Stulberg $(\mathrm{r}=0.70)$.

According to a combined assessment of the LP with the age at onset, Herring et al, ${ }^{3}$ reported that the LP classification gave a reliable prediction when age of more or less than nine years was introduced as a parameter. They found that LP group B showed a good outcome in patients who were under nine years at the time of onset (92\% Stulberg class I and II, $8 \%$ class III results), but a less favourable outcome in patients who were over nine years at onset $(30 \%$ Stulberg class II, $50 \%$ class III and $20 \%$ class IV results). Gigante et $\mathrm{al}^{10}$ also reported that the LP classification became prognostic when age was considered.

In the active stage of Perthes' disease, the possibility exists that inaccuracies occur in AP and lateral radiographs due to hip spasm. As flexion and abduction contracture can 
exert great influence on the PP classification based on lateral radiographs, it is necessary to take separate films of the right and left sides in order to compare symmetrically. In summary, our study shows that accurate prediction of outcome is not possible with the LP classification alone for patients classified into group B, but the CPS system does allow an accurate prediction of the radiological outcome for these patients.

The authors thank Doctors Y. Takahashi and S. Yamakawa for assistance with iner- and intra-observer reliability, Dr M. Kamegaya, Dr McQuire and Ms C. McCowan for reviewing the manuscript and Ms A. Yoshida for technical support.

No benefits in any form have been received or will be received from any commercial party related directly or indirectly to the subject of this article.

\section{References}

1. Catterall A. The natural history of Perthes' disease. J Bone Joint Surg [Br] 1971; 53-B:37-53.

2. Salter RB, Thompson GH. Legg-Calvé-Perthes disease: the prognostic significance of the subchondral fracture and a two-group classification of the femoral head involvement. J Bone Joint Surg [Am] 1984;66-A:479-89.

3. Herring JA, Neustadt JB, Williams JJ, Early JS, Browne RH. The lateral pilla classification of Legg-Calvé-Perthes disease. J Pediatr Orthop 1992;12:143-50.

4. O'Garra JA. The radiographic changes in Perthes' disease. J Bone Joint Surg $[\mathrm{Br}]$ 1959;41-B:465-76

5. Mukherjee A, Fabry G. Evaluation of the prognostic indices in Legg-Calvé-Perthes disease: statistical analysis of 116 hips. J Pediatr Orthop 1990;10:153-8.

6. Christensen F, Søballe K, Ejsted R, Luxhøj. The Catterall classification of Perthes disease: an assessment of reliability. J Bone Joint Surg [Br] 1986;68-B:614-15.
7. Hardcastle PH, Ross R, Hamalainen M, Mata A. Catterall grouping of Perthes disease: an assessment of observer error and prognosis using the Catterall classification. J Bone Joint Surg [Br] 1980;62-B:428-31.

8. Ritterbusch JF, Shantharam SS, Gelinas C. Comparison of lateral pillar classification and Catterall classification of Legg-Calvé-Perthes disease. J Pediatr Orthop 1993;13:200-2.

9. Ismail A, Macnicol MF. Prognosis in Perthes' disease: a comparison of radiological predictors. J Bone Joint Surg [Br] 1998;80-B:310-14

10. Gigante C, Frizziero P, Turra S. Prognostic value of Catterall and Herring classification in Legg-Calvé-Perthes disease: follow-up to skeletal maturity of 32 patients. $J$ Pediatr Orthop 2002:22:345-9.

11. Song HR, Lee SH, Na JB, et al. Comparison of MRI with subchondral fracture in the evaluation of extent of epiphyseal necrosis in the early stage of Legg-Calvé-Perthes disease. J Pediatr Orthop 1999;19:70-5.

12. Akazawa H, Miyake Y, Nagasawa F. Posterior pillar classification of Perthes' disease. J Jpn Paed Orthop Ass 2000;9:212-15.

13. Farsetti P, Tudisco C, Caterini R, Potenza V, Ippolito E. The Herring lateral pilla classification for prognosis in Perthes' disease. J Bone Joint Surg [Br] 1995;77-B:739-42.

14. Podeszwa DA, Stanitski CL, Stanitski DF, Woo R, Mendelow MJ. The effect of pediatric orthopaedic experience on interobserver and intraobserver reliability of the Herring lateral pillar classification of Perthes disease. J Pediatr Orthop 2000;20: $562-5$

15. Stulberg SD, Cooperman DR, Wallensten R. The natural history of Legg-CalvéPerthes disease. J Bone Joint Surg [Am] 1981;63-A:1095-108.

16. Sugioka Y, Katsuki I, Hotokebuchi T. Transtrochanteric rotational osteotomy of the femoral head for treatment of osteonecrosis: follow-up statistics. Clin Orthop 1982;169:115-26.

17. Cohen J. A coefficient of agreement for nominal scales. Educat and Psychol Measure 1960;20:37-46.

18. Cohen J. Weighted kappa: nominal scale agreement with provision for scaled disagreement or partial credit. Psychol Bull 1968;70:213-20.

19. Landis JR, Koch GG. The measurement of observer agreement for categorical data. Biometrics 1977;33:159-74 